\title{
A Retrospective Study of Invasive Fungal Disease Treated by High Dosage Micafungin Therapy VS Standard Micafungin Therapy Among Patients with Hematologic Diseases.
}

Jia Li ( $\square$ lijia050702@163.com )

Shengjing Hospital of China Medical University

Ying Lv

Shengjing Hospital of China Medical University

Guojun Zhang

Shengjing Hospital of China Medical University

Rong $\mathrm{Hu}$

Shengjing Hospital of China Medical University

Hong-Tao Wang

Shengjing Hospital of China Medical University

\section{Research Article}

Keywords: micafungin, invasive fungal disease, safety, efficacy, hematologic disease

Posted Date: June 1st, 2021

DOI: https://doi.org/10.21203/rs.3.rs-560720/v1

License: (c) (i) This work is licensed under a Creative Commons Attribution 4.0 International License.

Read Full License 


\section{Abstract}

Background: The standard (150mg/day) micafungin treatment regimen, is an effective and well tolerated empirical therapy for invasive fungal disease (IFD) among hematologic patients; however, experience with high dosage (200-300 mg/day) micafungin treatment is limited. We retrospectively evaluated both safety and effectiveness of micafungin as an empirical antifungal therapy via standard and high dosage treatments for IFD.

Methods: Patients were considered to be qualified for this analysis if they were proven, probable, or possible diagnosed as IFD. Patients without any previous exposure to an echinocandin were included; for those who changed treatment dosages and had previously received another systemic therapy except fluconazole for prevention were excluded. Safety and effectiveness were evaluated in all participated patients. IFD was diagnosed and classified according to standards defined by both European Organization for Research and Treatment of Cancer / Invasive Fungal Infections Cooperative Group, and the National Institute of Allergy and Infectious Diseases Mycoses Study Group Consensus Group.

Results: Totally 72 hematologic patients participated this study. 45 patients were received a standard (150mg/day)micafungin treatment, and 27 patients were received a high dosage (200$300 \mathrm{mg} /$ day)micafungin treatment. Neutropenia status were similar between two groups $(37.78 \%$ and $33.33 \%$ ), so were other underlying risk factors. The mean duration of therapy were 15.27 days (Range: 747 days) in standard micafungin group, and 14.96 days (Range: 7-51 days) in high dosagemicafungin group. Drug related adverse events (AEs) occurred in $11.11 \%$ of patients received the standard regimen, and $14.81 \%$ of patients received the high dosage regimen (difference, $3.70 \% ; 95 \%$ confidence interval [CI], $-5.75 \%$ to $10.89 \%$; statistically insignificant). The most common drug related AEs in the standard and high dosage treatment groups were liver dysfunction. Only 1 patient discontinued standard micafungin treatment, because liver function test value is 5 times greater than the upper limit of normal value. $86.67 \%$ of patients from standard treatment group, and $88.89 \%$ of patients from high dosage treatment group had favorable overall responses (difference, $2.22 \% ; 95 \% \mathrm{Cl},-4.94 \%$ to $8.64 \%$; statistically insignificant).

Conclusion: Both micafungin dosing treatments were effective, and well tolerated among hematologic patients with invasive fungal infection. Therefore, there hasn't arisen any safety concern of high dosage(200-300mg/day) micafungin treatment.

\section{Background}

Invasive fungal disease (IFD), such as Candida and Aspergillus species, has caused significant morbidity and mortality among hematologic patients in the recent years [1, 2]. Empirical antifungal treatments are used to reduce its consequences. Micafungin is an echinocandin antifungal agent with potent activity against most species of Candida and Aspergillus [3, 4]. Prior studies had demonstrated that micafungin at a dosage of $100 \mathrm{mg} /$ day was as effective as liposomal amphotericin B [5] and voriconazole[6], and 
superior toitraconazole[7] for the treatment of invasive candidiasis. For persistent febrile neutropenia, micafungin as an empirical antifungal therapy did not appear to differ significantly from caspofungin, in terms of safety profile and efficacy among adult patients [8]. Micafungin at a dosage of $150 \mathrm{mg} /$ day has been effective and well tolerated as an empirical therapy among hematologic patients with IFD [4]. Although some studies show that micafungin dosages exceeding $150 \mathrm{mg} /$ day are safe and well tolerated [9], the efficacy and safety of micafungin at higher dosage have not been fully investigated. Therefore, a retrospective study, mainly focused on the comparison of standard $(150 \mathrm{mg} /$ day) with high dosage (200$300 \mathrm{mg} /$ day) treatment in hematologic patients with IFD, had been conducted from January 2013 until December 2017 in Shengjing Hospital of China Medical University, in order to evaluate the safety, tolerability, and efficacy of micafungin among hematologic patients with IFD.

\section{Methods}

\section{Patient selection}

For hematologic patients above age of 14 , who also received micafungin therapy were considered as eligible candidates. The decisive factors for patients to be eventually included for this analysis were: they were proven, probable, or possible diagnosed as IFD. Proven IFD is defined as demonstration of fungal elements in infected tissue for most conditions irrespective of host factors or clinical features. Probable IFD requires a host factor, clinical features, and mycological evidence. Possible IFD includes cases with appropriate host factors and sufficient clinical evidence but no mycological support [10]. Patients without previous exposure to an echinocandin were included. Those who had changed treatment dosages, or previously received other systemic antifungal therapies for IFD (except fluconazole for prevention) were excluded. Safety and effectiveness were evaluated in all participated patients. IFD was diagnosed and classified according to standards defined by European Organization for Research and Treatment of Cancer/Invasive Fungal Infections Cooperative Group, and the National Institute of Allergy and Infectious Diseases Mycoses Study Group Consensus Group criteria [10-14].

\section{Safety and efficacy evaluation}

Medical records of all participated patients were reviewed from both clinical and microbiologic perspectives. Micafungin dosages, length of therapy, and drug-related AEs were also documented. Baseline IFD was defined as a case diagnosed within 2 days of echinocandin initiation; breakthrough IFD occurred more than 3 days after initiation of treatment.

Patients were closely monitored on daily basis for potential AEs during micafungin therapy. All the drug related AEs which could potentially led to discontinuation of micafungin treatment, such as vital signs, blood routine, liver function and renal function, were documented and monitored for safety purposes.

Efficacy analyses were conducted for following cases: treatment of any baseline IFD successfully treated by micafungin therapy, absence of any breakthrough IFD during/within 7 days after completion of therapy, and discontinuation of therapy due to drug-related AEs. 


\section{Statistical methods}

The primary hypothesis was that the high dosage micafungin treatment would be non-inferior to the standard treatment with respect to the development of significant drug-related AEs and efficacy. Noninferiority was defined by such that the upper limit of the 2-sided $95 \%$ confidence interval (Cl) for the treatment group difference, in term of percentage of patients with significant drug-related AEs or efficacy must be less than $15 \%$.

\section{Results}

\section{Baseline characteristics}

Among total of 72 qualified hematologic patients participated this study, 45 patients were in the standard (150mg/day) treatment group, and 27 patients in the high dosage (200-300 mg/day) treatment group. The two treatment groups were similar at study entry (Table 1). Such as, median age was 47 and 48 years old in the standard and high dosage micafungin treatment group; 28 out of 45 (62.22\%) and 17 out of $27(62.96 \%)$ patients in each group were males. Furthermore, the disease (leukemia) were same for both groups, neutropenia status were similar (37.78\% and $33.33 \%)$, and the main underlying risk factors were same between these two groups. 
Table 1

Patients Characteristics

\begin{tabular}{|c|c|c|}
\hline Parameter & $\begin{array}{l}\text { Micafungin } 150 \mathrm{mg} / \mathrm{d} \\
(\mathrm{n}=45)\end{array}$ & $\begin{array}{l}\text { Micafungin } 200-300 \mathrm{mg} / \mathrm{d} \\
(\mathrm{n}=27)\end{array}$ \\
\hline \multicolumn{3}{|l|}{ Gender } \\
\hline Male & $28(62.22 \%)$ & $17(62.96 \%)$ \\
\hline Female & 17 (37.78\%) & $10(37.04 \%)$ \\
\hline \multicolumn{3}{|l|}{ Age,years } \\
\hline Mean \pm SD & $46.93 \pm 18.31$ & $47.33 \pm 17.65$ \\
\hline Median (range) & $47(15-86)$ & $48(14-75)$ \\
\hline \multicolumn{3}{|l|}{ Underlying Disease } \\
\hline leukemia & 32 (71.11\%) & $18(66.67 \%)$ \\
\hline MDS & $1(2.22 \%)$ & $3(11.11 \%)$ \\
\hline Lymphoma & $4(8.89 \%)$ & $1(3.70 \%)$ \\
\hline MM & $6(13.33 \%)$ & $3(11.11 \%)$ \\
\hline AA & $2(4.44 \%)$ & $2(7.41 \%)$ \\
\hline \multicolumn{3}{|l|}{ Neutropenia status } \\
\hline 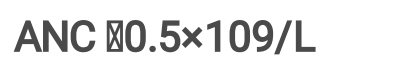 & 17 (37.78\%) & $7(25.93 \%)$ \\
\hline$A N C \geq 0.5 \times 109 / L$ & $28(62.22 \%)$ & $20(74.07 \%)$ \\
\hline \multicolumn{3}{|l|}{ Risk factors } \\
\hline Diabetes mellitus & $5(11.11 \%)$ & $2(7.41 \%)$ \\
\hline Immuno-suppression & $2(4.44 \%)$ & $1(3.70 \%)$ \\
\hline Previous IFD & $1(2.22 \%)$ & $2(7.41 \%)$ \\
\hline Hormone therapy & $9(20 \%)$ & $7(25.93 \%)$ \\
\hline
\end{tabular}

\section{Diagnosis and treatment duration}

In the standard ( $150 \mathrm{mg} /$ day) treatment group: 3 out of 45 patients $(6.67 \%)$ were proven diagnosed, 17 out of 45 patients (37.78\%) were probable diagnosed, and 25 out of 45 patients (55.56\%) were possible diagnosed as IFD. While in the high dosage (200-300 mg/day) treatment group, the number of proven, probable and possible diagnosed as IFD patients among 27 participated patients were 1 (3.70\%), 14 (51.85\%) and $12(44.44 \%)$, respectively. 
The mean duration of micafungin therapy was almost identical for both treatment groups, 15.27 days [range, 7-47 days] for the standard (150mg/day) treatment group, and 14.96 days [range, 7-51 days] for the high dosage (200-300mg/day) treatment group.

\section{Safety and tolerability}

Severe drug-related AEs were reported in 2 out of 45 patients $(4.44 \%)$ in the standard $(150 \mathrm{mg} /$ day) treatment group, and 1 out of 27 patients $(3.70 \%)$ in the high dosage $(200-300 \mathrm{mg} / \mathrm{day})$ treatment group. The observed difference between the treatment groups was $-0.74 \%$, with a $95 \% \mathrm{Cl}$ of $-4.78-4.12 \%$, which fulfilled the predefined criterion for non-inferiority. The incidences of discontinuations of micafungin therapy because of drug-related clinical AEs (2.22\% VS 0\%) were similar between the standard (150mg/day) and high dosage (200-300mg/day) treatment groups. The most common drug-related laboratory AEs in both treatment groups were increased alanine aminotransferase (ALT), and aspartate aminotransferase (AST). None of the drug-related laboratory events were serious or led to discontinuation of micafungin treatment. The overall rates of clinical and laboratory AEs, regardless of causality, were also similar (11.11\% VS $14.81 \%$ ) between the standard and high dosage treatment groups (difference, 3.70\%; 95\% Cl, $-5.75-10.89 \%$ ), respectively (Table 2 ).

Table 2

Drug Related Adverse Events

\begin{tabular}{|lll|}
\hline Drug Related Adverse Events N (\%) & $\begin{array}{l}\text { Micafungin } \\
150 \mathrm{mg} / \mathrm{d}(\mathrm{n}=45)\end{array}$ & Micafungin 200-300mg/d ( $=27)$ \\
\hline ALL & $5(11.11)$ & $4(14.81)$ \\
\hline Severe & $2(4.44)$ & $1(3.70)$ \\
\hline Leading to discontinue & $1(2.22)$ & $0(0)$ \\
\hline ALT increased & $4(8.89)$ & $2(7.41)$ \\
\hline AST increased & $5(11.11)$ & $3(11.11)$ \\
\hline Cr increased & $0(0)$ & $1(3.70)$ \\
\hline
\end{tabular}

Furthermore, there were no differences in terms of parameters such as blood pressure, pulse rate, body weight, routine blood count, and renal function before and after micafungin therapy, in both standard (150mg/day) and high dosages (200-300mg/day) treatments, respectively (Table 3 ). 
Table 3

Safety

\begin{tabular}{|lllll|}
\hline Parameter & \multicolumn{2}{l}{$\begin{array}{l}\text { Micafungin 150mg/d } \\
(n=45)\end{array}$} & $\begin{array}{l}\text { Micafungin 200-300mg/d } \\
(n=27)\end{array}$ & \\
\cline { 2 - 5 } & Before Treatment & After Treatment & Before Treatment & After Treatment \\
\hline Blood pressure & & & & \\
\hline Systolic BP & $115.67 \pm 12.25$ & $115.28 \pm 11.92$ & $113.96 \pm 12.50$ & $116.40 \pm 14.23$ \\
\hline Diastolic BP & $72.23 \pm 8.76$ & $72.50 \pm 9.21$ & $70.68 \pm 9.92$ & $69.52 \pm 9.38$ \\
\hline Pulse & $93.82 \pm 14.14$ & $88.79 \pm 16.44$ & $94.59 \pm 12.45$ & $84.11 \pm 11.79$ \\
\hline Body weight & $63.52 \pm 11.41$ & $62.45 \pm 11.78$ & $56.54 \pm 8.43$ & $56.52 \pm 8.09$ \\
\hline Routine Blood Count & & & & \\
\hline WBC (109/L) & $10.68 \pm 38.34$ & $4.40 \pm 4.93$ & $4.83 \pm 3.52$ & $4.67 \pm 2.99$ \\
\hline Neutrophil (109/L) & $2.79 \pm 5.78$ & $2.16 \pm 2.18$ & $3.23 \pm 2.70$ & $2.79 \pm 2.34$ \\
\hline PTL (109/L) & $71.44 \pm 107.53$ & $97.54 \pm 117.57$ & $83.85 \pm 80.68$ & $105.97 \pm 78.39$ \\
\hline Liver function & & & & \\
\hline ALT (U/L) & $35.23 \pm 39.36$ & $30.72 \pm 29.49$ & $65.22 \pm 183.04$ & $40.26 \pm 42.96$ \\
\hline AST (U/L) & $29.56 \pm 30.24$ & $45.96 \pm 90.24$ & $55.48 \pm 143.51$ & $36.07 \pm 33.14$ \\
\hline Creatine (ug/dl) & $59.60 \pm 19.99$ & $58.44 \pm 17.48$ & $55.40 \pm 16.82$ & $58.88 \pm 21.15$ \\
\hline
\end{tabular}

\section{Efficacy}

The efficacy of all 72 participated hematologic patients was evaluated. By the end of micafungin therapy, an $86.67 \%$ of favorable clinical response occurred in the standard (150mg/day) treatment group, and $88.89 \%$ of the high dosage $(200-300 \mathrm{mg} /$ day) treatment group, with a $95 \% \mathrm{Cl}$ of $-4.94-8.64 \%$. A favorable image exam response occurred for $55.56 \%$ of patients in the standard $(150 \mathrm{mg} /$ day)treatment group, and $59.26 \%$ of patients in the high dosage (200-300 mg/day) treatment group. Response differences between the two treatment groups were insignificant (Table 4). 
Table 4

Efficacy

\begin{tabular}{|c|c|c|c|c|}
\hline \multirow[t]{2}{*}{ Parameter } & \multicolumn{2}{|c|}{$\begin{array}{l}\text { Micafungin } 150 \mathrm{mg} / \mathrm{d} \\
(\mathrm{n}=45)\end{array}$} & \multicolumn{2}{|c|}{$\begin{array}{l}\text { Micafungin } 200-300 \mathrm{mg} / \mathrm{d} \\
(\mathrm{n}=27)\end{array}$} \\
\hline & $\begin{array}{l}\text { Before treatment } \\
(n, \%)\end{array}$ & $\begin{array}{l}\text { Remission cases } \\
(n, \%)\end{array}$ & $\begin{array}{l}\text { Before treatment } \\
(n, \%)\end{array}$ & $\begin{array}{l}\text { Remission cases } \\
(n, \%)\end{array}$ \\
\hline \multicolumn{5}{|l|}{ Symptoms } \\
\hline Fever & $36(80.00)$ & \multirow[t]{4}{*}{$39(86.67)$} & 23 (85.19) & \multirow[t]{4}{*}{$24(88.89)$} \\
\hline Caugh & $25(55.56)$ & & 19 (70.37) & \\
\hline Sputum & 21 (46.67) & & $13(48.15)$ & \\
\hline Dispnea & $11(24.44)$ & & 5 (18.52) & \\
\hline \multicolumn{5}{|l|}{ Image exam } \\
\hline X-ray & $3(6.67)$ & \multirow[t]{3}{*}{$25(55.56)$} & $1(3.70)$ & \multirow[t]{3}{*}{$16(59.26)$} \\
\hline CT & 37 (82.22) & & 21 (77.78) & \\
\hline $\begin{array}{l}\text { High- } \\
\text { resolution CT }\end{array}$ & $5(11.11)$ & & 5 (18.52) & \\
\hline
\end{tabular}

Furthermore, three patients $(3 / 45)$ were detected fungal infection by blood culture in standard (150mg/day) group, while one patient (1/27) was detected in high dosage (200-300mg/day) group, and all the blood cultures turned negative after treatments. Four patients $(4 / 45)$ were detected fungal infection by sputum culture in standard $(150 \mathrm{mg} /$ day) treatment group, and two patients $(2 / 27)$ were detected in high dosage (200-300mg/day) treatment group. Totally three patients in high dosage (200$300 \mathrm{mg} /$ day) treatment group took BALF examination, but fungal were not detected. However, no patients from standard (150mg/day) treatment group took BALF exams. G and GM test results are shown in Fig. 1.

\section{Discussion}

IFD is a significant cause of morbidity and mortality for hematologic patients. The risk of IFDs is dramatically increased in patients with hematologic disease, who are undergoing intensive chemotherapy or stem cell transplantation, as well as in other immune compromised populations [15]. The most commonly identified fungal species associated with IFD are Candida, Aspergillus, Cryptococcus and Pneumocystis[16]. The second marketed echinocandinmicafungin was approved in 2005 for the treatment of esophageal candidiasis, and prophylaxis of invasive Candida infections in patients who are undergoing hematopoietic stem cell transplantation. A retrospective multicenter observation study showed that 159 patients who received allogeneic hematopoietic stem cell transplantation,also received $50 \mathrm{mg} /$ day of micafungin to prevent fungal infection, with an effective rate of $94.9 \%[17]$. Another 
retrospective study showed that the application of micafungin in the prevention of fungal infection, in patients with hematological diseases receiving chemotherapy or immunosuppressive therapy, also has good efficacy and safety[18].At present, theindications of micafunginusage have been expanded to include candidemia, acute disseminated candidiasis, Candida abscesses, and peritonitis. Similar to other echinocandins, it is fungicidal against Candida species, including those that are polyene and azole resistant and fungistatic against Aspergillus species [3].

Prior studies had demonstrated that micafungin at standard dosage $(150 \mathrm{mg} /$ day $)$ in adults was effective, and well tolerated as an empirical therapy for IFD patients with hematologic diseases [4]. The dosage can be increased up to $300 \mathrm{mg} /$ day in the case of severe fungal infections;however, the efficacy and safety of micafungin at high dosage have not been fully investigated. Therefore, we compared the standard (150mg/day) micafungin treatment with high dosage (200-300mg/day) treatment among hematologic patients with IFD.

In our study, the baseline characteristics of standard and high dosage micafungin treatment groups were comparable. The status of safety and tolerability were similar between two groups, as well as the number of AEs occurrences. Severe AEs and discontinuation of therapy due to AEs were rare cases. It suggested that the high dosage micafungin therapy was a safe and well tolerated treatment for hematologic patients. These findings were consistent with the results from the previous studies that also led to the safety profile of high dosage micafungin[9]. Furthermore, the efficacy of two treatment groups were also similar, the standard treatment group was not inferior to the high dosage treatment group. This conclusion should be noticeable. Several studies have indicated that the standard dosage is sufficient to treat Candida infections [5, 19]; however, a pharmacokinetic pharmacodynamic analysis showed that a 200-300mg daily dosage micafungin treatment for Aspergillus infections is associated with favorable outcomes [20]. In our study, only 3 out of 45 patients (6.67\%) in standard treatment group were diagnosed as candidiasis, and 1 out of $27(3.70 \%)$ patient in high dosage treatment group was diagnosed as Aspergillus. Most patients from both treatment groups weren't able to be diagnosed $(55.56 \%$ for standard group, and $44.44 \%$ for high dosage group). There wasn't any mycological evidence shown the type of IFD. Standard micafungin therapy is likely suitable as an empirical treatment for IFD, especially Candida infections; while high dosage treatment only should be practiced if evidences of Aspergillus infections have been indicated.

\section{Conclusion}

Both standard and high dosage micafungin treatments were effective and well tolerated in patients with invasive fungal infection. No safety concerns have been found for micafungin treatment at a dosage of $200-300 \mathrm{mg} /$ day.

\section{Abbreviations}

IFD: invasive fungal disease 
AEs: adverse events

Cl: confidence interval

ALT: alanine aminotransferase

AST: aspartate aminotransferas3

\section{Declarations}

\section{Ethics approval and consent to participate}

The ethics committee of Shengjing Hospital of China Medical University has reviewed the study and has determined that this is a retrospective study and the design is scientifically and is up to the ethics standards. The ethics committee thus approve the study. Written informed consent was waived due to retrospective study design. [No. 2021PS043K].

\section{Consent for publication}

Not applicable.

\section{Availability of data and materials}

The datasets used and/or analyzed during the current study are available from the corresponding author on reasonable request.

\section{Competing interests}

The authors declare that they have no competing interests.

\section{Funding}

This work was supported by grants from National Natural Science Foundation of China (81600115).

\section{Authors' contributions}

JL and HW designed the study and drafted the manuscript. YL, GZ, and RH collected the patient information and analyzed the data. All authors were involved in finalizing the manuscript and approved the final version of the manuscript. 


\section{Acknowledgments}

Not applicable.

\section{Authors' Information}

${ }^{1}$ Department of Hematology, Shengjing Hospital, China Medical University, No.39 Huaxiang Road, Tiexi District, Shenyang, Zip code: 110022, Liaoning China.

\section{References}

1. Blyth CC, Gilroy NM, Guy SD, et al. Consensus guidelines for the treatment of invasive mould infections in haematological malignancy and haemopoietic stem cell transplantation, 2014. Internal medicine journal. 2014; 44: 1333-49.

2. Chen $\mathrm{K}$, Wang Q, Pleasants RA, et al. Empiric treatment against invasive fungal diseases in febrile neutropenic patients: a systematic review and network meta-analysis. BMC infectious diseases. 2017; 17: 159.

3. de la Torre P, Reboli AC. Micafungin: an evidence-based review of its place in therapy. Core evidence. 2014; 9: 27-39.

4. Yamaguchi M, Kurokawa T, Ishiyama K, et al. Efficacy and safety of micafungin as an empirical therapy for invasive fungal infections in patients with hematologic disorders: a multicenter, prospective study. Annals of hematology. 2011; 90: 1209-17.

5. Jean FT, Guy L, Noël M, et al. Treatment of invasive fungal infections in intensive care units with micafungin: The MYRIADE study. Mycoses. 2020; 63: 443-51.

6. Oyake T, Kowata S, Murai K, et al. Comparison of micafungin and voriconazole as empirical antifungal therapies in febrile neutropenic patients with hematological disorders: a randomized controlled trial. European journal of haematology. 2016; 96: 602-9.

7. Jeong SH, Kim DY, Jang JH, et al. Efficacy and safety of micafungin versus intravenous itraconazole as empirical antifungal therapy for febrile neutropenic patients with hematological malignancies: a randomized, controlled, prospective, multicenter study. Annals of hematology. 2016; 95: 337-44.

8. Kubiak DW, Bryar JM, McDonnell AM, et al. Evaluation of caspofungin or micafungin as empiric antifungal therapy in adult patients with persistent febrile neutropenia: a retrospective, observational, sequential cohort analysis. Clinical therapeutics. 2010; 32: 637-48.

9. Yamazaki S, Nakamura F, Yoshimi A, et al. Safety of high-dose micafungin for patients with hematological diseases. Leukemia \& lymphoma. 2014; 55: 2572-6.

10. De Pauw B, Walsh TJ, Donnelly JP, et al. Revised definitions of invasive fungal disease from the European Organization for Research and Treatment of Cancer/Invasive Fungal Infections Cooperative Group and the National Institute of Allergy and Infectious Diseases Mycoses Study 
Group (EORTC/MSG) Consensus Group. Clinical infectious diseases : an official publication of the Infectious Diseases Society of America. 2008; 46: 1813-21.

11. Walsh TJ, Anaissie EJ, Denning DW, et al. Treatment of aspergillosis: clinical practice guidelines of the Infectious Diseases Society of America. Clinical infectious diseases : an official publication of the Infectious Diseases Society of America. 2008; 46: 327-60.

12. Pappas $P G$, Kauffman $C A$, Andes $D$, et al. Clinical practice guidelines for the management of candidiasis: 2009 update by the Infectious Diseases Society of America. Clinical infectious diseases : an official publication of the Infectious Diseases Society of America. 2009; 48: 503-35.

13. Maertens $\mathrm{J}$, Marchetti $\mathrm{O}$, Herbrecht R, et al. European guidelines for antifungal management in leukemia and hematopoietic stem cell transplant recipients: summary of the ECIL 3-2009 update. Bone marrow transplantation. 2011; 46: 709-18.

14. Castagna L, Bramanti S, Sarina B, et al. ECIL 3-2009 update guidelines for antifungal management. Bone marrow transplantation. 2012; 47: 866.

15. Deng Q, Lv HR, Lin XM, et al. Empirical antifungal treatment for diagnosed and undiagnosed invasive fungal disease in patients with hematologic malignancies. Current medical research and opinion. 2017: 1-8.

16. Schmiedel Y, Zimmerli S. Common invasive fungal diseases: an overview of invasive candidiasis, aspergillosis, cryptococcosis, and Pneumocystis pneumonia. Swiss medical weekly. 2016; 146: w14281.

17. Cristina LS, David V, Valle G, et al. Use of micafungin as antifungal prophylasix in patients undergoing allogeneic hematopoietic stem cell transplantation (allo-HSCT) in Spain (GETH-MIC). Revista Española de Quimioterapia. 2020; 33: 110-5.

18. Teresa V, Lourdes V, Juan MB, et al. Micafungin as antifungal prophylasix in non-transplanted haemotological patients. Revista Española de Quimioterapia. 2020; 33: 44-8.

19. Pappas PG, Rotstein CM, Betts RF, et al. Micafungin versus caspofungin for treatment of candidemia and other forms of invasive candidiasis. Clinical infectious diseases : an official publication of the Infectious Diseases Society of America. 2007; 45: 883-93.

20. Ikawa K, Nomura K, Morikawa N, et al. Assessment of micafungin regimens by pharmacokineticpharmacodynamic analysis: a dosing strategy for Aspergillus infections. The Journal of antimicrobial chemotherapy. 2009; 64: 840-4.

\section{Figures}




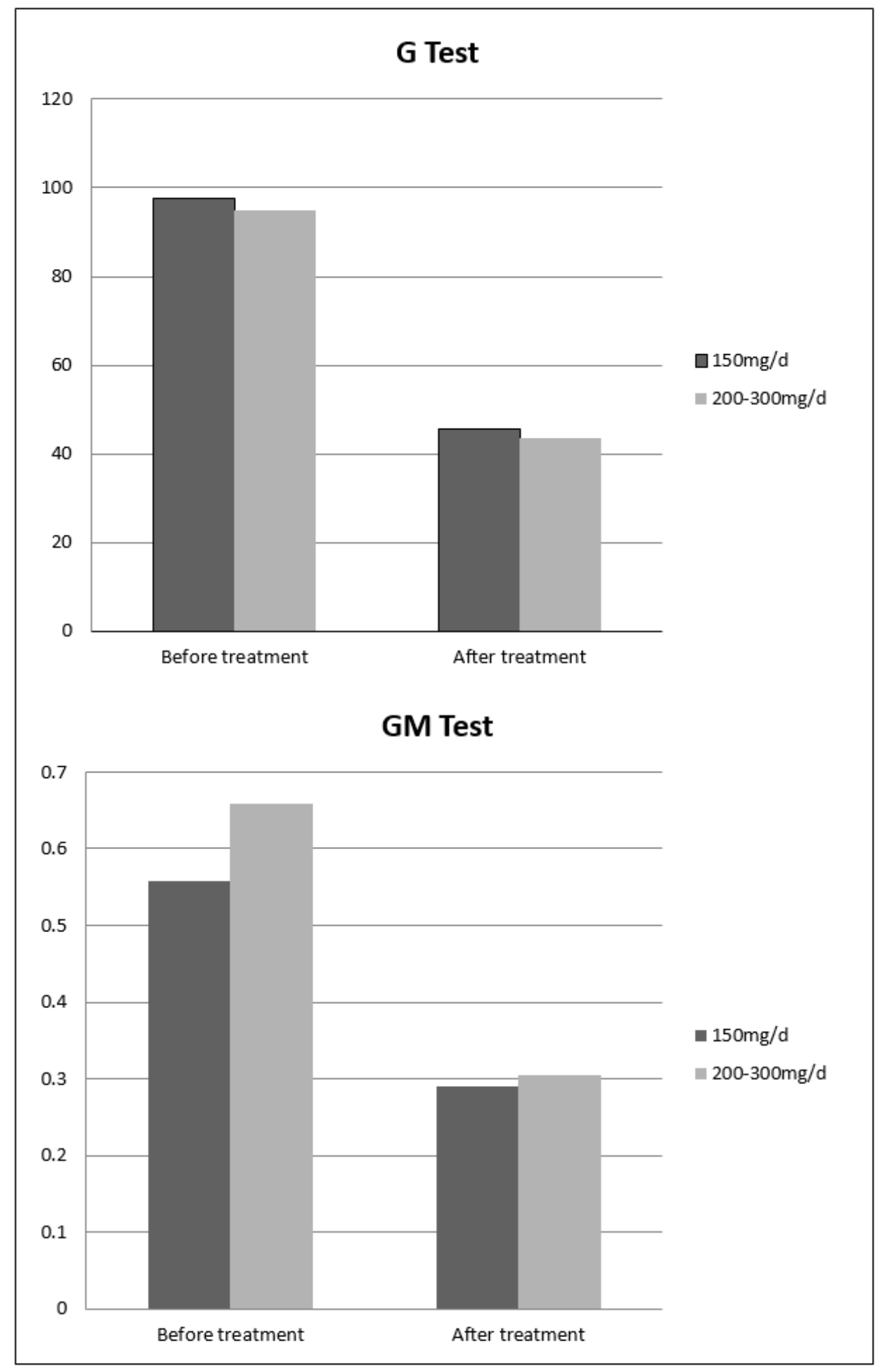

Figure 1

G and GM Test of $150 \mathrm{mg} / \mathrm{d}$ and $200-300 \mathrm{mg} / \mathrm{d}$ Treatments 